\title{
On the Experience of Treating Obese Type 2 Diabetes Mellitus
}

\author{
Dan Bi ${ }^{1}$, Huaizhen $\mathrm{Liu}^{2}, *$ \\ ${ }^{1}$ Anhui University of Chinese Medicine, Hefei 230012, China \\ ${ }^{2}$ Department of Endocrinology, Geriatrics Center, The First Affiliated Hospital of Anhui University of Traditional Chinese Medicine, \\ Hefei 230000, China \\ *Correspondence Author
}

\begin{abstract}
Obesity type 2 diabetes is an illusion of the spleen in TCM. Professor Liu Huaizhen believes that the origin of obesity type 2 diabetes is spleen deficiency, which is mainly characterized by deficiency of temper, and is marked by evil and solid. It is mainly characterized by internal obstruction of dampness-heat phlegm and blood stasis, which is caused by the combination of deficiency and solid. Therefore, "spleen deficiency damp-heat" is the main pathogenesis and important link of the occurrence and development of obese type 2 diabetes. Professor Liu believed that the treatment of the disease should first strengthen the spleen, supplemented by clearing away dampness and heat, taking into account the reconciliation of sanjiao, accompanied by phlegm and stasis. It has a good clinical effect and provides a good idea for TCM prevention and treatment of obesity type 2 diabetes.
\end{abstract}

Keywords: Liu Huaizhen, Obesity, Type 2 diabetes mellitus, Spleen deficiency and damp-heat, Experience of famous doctors.

\section{Introduction}

Modern medicine considers obesity as a chronic metabolic disease with excessive accumulation or abnormal distribution of body fat, which has become a public health problem of global human importance[1]. Type 2 diabetes mellitus (T2DM) and obesity are both endocrine metabolic diseases, and obesity can lead to endothelial function damage, which causes insulin resistance, and insulin resistance is the core pathogenesis of T2DM, and they are closely related. A study[2] showed that the vast majority of patients with T2DM are obese type 2 diabetic. Obese type 2 diabetes can lead to a variety of fatal and disabling diseases such as stroke, cardiovascular disease, and depression[3]. This disease is a common disease treated by Professor Liu Huaizhen, who believes that this disease belongs to the "spleen disease" in Chinese medicine, and the pathogenesis is based on spleen deficiency, which results in internal congestion of dampness and heat, and the onset of the disease is caused by the stagnation of phlegm and stagnation of evil into sugar turbidity over time[4]. Therefore, Professor Liu's treatment is mostly based on the theory of "spleen deficiency, dampness and heat", and he uses prescriptions, investigates the mechanism of legislation, and selects medicines, which is always effective. I have the honor to consult with him and have benefited greatly. I would like to compile the academic thoughts of Professor Liu on the treatment of this disease for the benefit of readers.

\section{Learn from the Past and the Present, Examine the Evidence and Seek the Opportunity}

Type 2 diabetes belongs to the category of "quenching thirst" in Traditional Chinese medicine. Different from typical "three more and one less" diabetes patients, obese type 2 diabetes patients are mostly manifested by obesity, sweet mouth, bitter mouth, dry mouth, fatigue and weakness, and are mostly characterized by increased body mass index, waist-to-hip ratio and blood sugar found in physical examination.
According to The Treatise on Strange Diseases, "The spillage of the five senses is also called an illusion of the spleen. The fat hair is also... Fat people will be internal heat, sweet people will be full, thus causing qi to overflow, and hair to quenchthirst." Therefore, "Hate of spleen" can correspond to modern obese type 2 diabetes patients. Professor Liu Huaizhen believes that obesity type 2 diabetes first lies in spleen deficiency, secondary to damp-heat, phlegm and blood stasis mutual knot, tired of the whole body viscera and disease.

\subsection{Spleen Deficiency is the Basis}

The spleen is the foundation of the posterior, the spleen and the stomach are weak and all diseases are born. "Drink into the stomach, overflowing essence, upward transmission in the spleen, the spleen disperses essence ..." Spleen qi deficiency, the inability to disperse water and grain essence, so that water into wet, grain into stagnation, wet stagnation to stay in the body, phlegm and turbidity internal obstruction and obesity. Su Wen - strange disease theory said: "spleen (disease)... eat more sweet and beautiful also ... turn into thirst". Fat hinders the stomach, sweet stagnation of the spleen. The obese body and the preference for thick, fatty and sweet foods are the important causes of its development, and diet hurts the stomach and fatigue hurts the spleen. If you eat unclean food, eat a lot of fat and sweet, food stagnates in the middle jiao, stagnates the stomach and hinders the spleen, the spleen and earth are congested, the spleen loses its health, phlegm and turbidity are generated inside, the blood and Qi do not run smoothly, phlegm and heat are congested and become full in the middle, full in the middle must have dampness and turbidity, gathering and generating dampness and heat, full in the middle and internal heat, and the fluid does not go up and develop into thirst. Professor Liu Huaizhen believes that the root cause of obesity type 2 diabetes is spleen deficiency. Therefore, due to diet and irregular life, obese type 2 diabetic patients suffer from internal deficiency combined with external evil, resulting in obesity, sweet mouth, dry mouth, fatigue and weakness, etc. 


\subsection{Damp-heat as a Symptom}

The treatise on warmth and fever says: "Spleen disease is damp-heat gas gathering with grain gas, fullness is on the pan". The spleen is not only hot, but also damp-heat, which is called damp-heat[5]. Such as the "miscellaneous evidence of the repertory": the beginning of the disease, all from ... damp-heat gas, depression into dry heat. The initial pathogenesis of thirst is the attack of damp-heat, and the onset of dryness over time. The spleen is the main transport and transformation, spleen deficiency, transport and transformation is not normal, easy to brew dampness, dampness is easy to block the qi, brew phlegm and dampness and obesity, dampness is not transformed, gathered for a long time into phlegm and heat, dampness and heat stop in the spleen and stomach, heat consumption and injury, the internal organs are not moistened and nourished, then thirst. Danxi Xinfa said: "damp depression is into heat, heat depression is into phlegm, phlegm depression is not blood ... six because of the disease also." Dampness and heat accumulate in the body, phlegm and dampness are contained, stagnation of qi and blood will become stasis, phlegm and stasis in the interior, with the qi lift and fall will be a series of various changes in evidence. It can be seen that phlegm and stasis are the important pathological products and the source of its variables. Professor Liu Huaizhen believes that damp-heat is the key pathogenic factor of obese type 2 diabetes, and phlegm-stasis is the root of its variant.

\section{Based on Spleen Deficiency and Damp-heat, the Treatment is to Explore the Mystery}

\subsection{Strengthen the Spleen, Benefit Qi, and Protect the Spleen and Stomach}

"Eliminate the spleen in the middle". The spleen and the stomach are on top of each other, dryness and dampness, dampness and heat are trapped in the spleen, the stomach will suffer from it, the spleen is the root of the posterior, the spleen and stomach injury and all diseases are born. Therefore, it is advisable to strengthen the spleen to help the spleen to transport, in order to treat its root. It is stated in "Treating the Middle Jiao like a balance, not a peace of uneasiness". Therefore, Prof. Liu Huaizhen pays special attention to protecting the spleen and stomach, and advocates that treatment of the spleen and stomach should focus on the balance of the middle jiao, so as to level the period and regulate the qi lifting and lowering function of the spleen and stomach, and the spleen and stomach will be at ease. In clinical practice, he usually uses Ginseng Ling Bai Zhu San and Er Chen Tang or Han Xia Di Xin Tang to add and subtract. Strengthen the spleen, benefit the qi, resolve dampness, elevate the clear and lower the turbidity, and regulate the qi flow, so that the disease will disappear. For those with poor appetite, aromatic and qi-regulating products such as patchouli and pelargonium should be added to help the spleen and stomach to function. The spleen and stomach belong to the earth, in the middle of the mediation, and the other four organs are more closely related, Liu Shi believes that dampness is connected to the spleen, the clinical evidence should pay great attention to regulating the spleen and stomach, to dry dampness to help each other, lifting and lowering the normal, in order to follow its nature.

\subsection{Distinguishing Between Dampness and Heat, Clearing and Promoting Dampness and Heat}

3.2.1 Clearing heat and relieving dampness where heat is more important than dampness

Dampness is contained in the spleen and stomach, which turns into heat over time and results in the evidence of damp-heat. Patients who have a strong Yang energy and a solid middle qi, the evil is transformed from heat, the patient often shows that heat is heavier than damp and lighter than damp, and the symptoms are often accompanied by obesity, bad taste in the mouth, dry mouth, bitter mouth and abdominal distention. The clinical manifestations are postprandial blood sugar and fasting blood sugar control are average. Treatment should be to clear heat and remove dampness at the same time. Liu Shi is good at using Ge Gen Scutellaria Tang as the basic formula for his treatment, taking its effect of clearing damp-heat, quenching thirst and raising fluid, helping damp-heat to be expelled from the body quickly. According to ancient books, Scutellaria baicalensis and Scutellaria baicalensis are good at treating elimination. Modern pharmacological studies have shown that Scutellaria baicalensis and Radix Scutellariae have hypoglycemic effects, and baicalein contained in Scutellaria baicalensis can agitate oxidative stress, significantly reduce oxygen free radicals in rats and improve islet function through antioxidant effects[6-7]; the scutellarin and scutellarin contained in Radix Scutellariae can repair pancreatic $\beta$-cells and improve insulin resistance[7]. Isoflavones are the main active substances contained in Pueraria lobata, which are good at lowering blood sugar, lowering blood lipids, anti-lipid peroxidation, accelerating liver fat metabolism, and reducing cardiovascular and cerebrovascular damage[8]. Some studies have shown[9] that Ge Gen Scutellaria Tang can treat obese type 2 diabetes by regulating the intestinal flora in the body and improving insulin resistance. Based on years of clinical experience, Liu Shi concluded that Ge Gen Scutellaria Tang can improve insulin function, accelerate glucolipid metabolism, and lower blood glucose.

\subsubsection{Dampness is heavier than heat in clearing dampness and} heat

Patients with weak yang energy and deficient middle qi often show that dampness is heavier than heat, and they often see obesity, sticky mouth, heavy head and body, stuffiness in the chest and epigastrium, weakness and tiredness, and poor appetite. Clinical manifestations are high postprandial blood glucose, but fasting blood glucose control is still possible. The treatment should be aromatizing dampness and taking into account clearing heat. Professor Liu Huaizhen makes good use of Ginseng and Atractylodes San combined with Huang Lian and Wen Gall Bladder Soup plus reduction. Studies have shown that Huang Lian Wen Bile Tang can improve blood lipid metabolism, counteract insulin resistance, and improve the patient's TCM symptoms[10]. As the ancient saying goes, "treating with orchids removes stale qi", Liu Shi often uses pelargonium, calamus, patchouli, and lotus leaves to awaken the spleen, resolve dampness, and cleanse sweet fat, followed by removing dampness and heat, and modern pharmacological studies have shown that pelargonium and other drugs[11] lower blood glucose by regulating glucolipid 
metabolism and inhibiting the activity of $\alpha$ glucosidase. The treatment of dampness is not its treatment, aromatic dampness products are more pungent, can help to wake up the spleen and eliminate turbidity, promote the smooth flow of Qi to clear heat and dampness. The clinical efficacy is very good when both glucose lowering and fat reduction are taken into account.

\subsection{Harmonize the Three Jiao, Divided and Eliminated}

"The evil of damp-heat, not from the table and into ...... Utah's Hejian treatment of thirst, to the three jiao points is also." The evil of damp heat is good at running, can diffuse the upper, middle and lower three jiao, stagnation in the three jiao, the pivot is not conducive, in turn, damp heat more, water and grain essence transmission and distribution is not regulated, and over time accumulate in the body, the formation of sugar turbidity and thirst. Therefore, the treatment should take into account the three jiao, qi line is wet move, wet go to the heat alone, wet and heat evil to make the split and eliminate. Liu Shi makes good use of San Ren Tang with addition and reduction to open the upper, smooth the middle, and permeate the lower in order to divide the channels and eliminate, effectively improving the patient's bitterness, stickiness, tiredness and weakness, and frequent urination. Some studies have shown[12-13] that San Ren Tang can significantly reduce blood glucose and alleviate obesity in obese type 2 diabetic patients by reducing the effect of body fat on insulin function, with good therapeutic effects. Liu Shi pointed out that the reconciliation of the three jiao, the removal of dampness will make the heat clear, so that sugar, lipids, turbidity and stasis are eliminated and removed, and the clinical results are quite good.

\subsection{Eliminate Phlegm and Eliminate Blood Stasis to Prevent its Change}

If spleen disease is not treated for a long time, it will accumulate in the internal organs, block the qi and blood, disturb the yin and yang, and then change evidence will arise. Dampness and heat trapped in the spleen and stomach, long time, then qi deficiency, pushing weak, phlegm and stasis within; blocking the qi, qi and blood is not smooth, then phlegm and stasis each other; phlegm and stasis blocking the spleen and stomach, vascular stasis, qi and blood stagnation, long time endanger the whole body organs and meridians, followed by stroke, edema, paralysis, gangrene and other changes. In the article "Relationship between TCM evidence and serum homocysteine and cystatin $\mathrm{C}$ in patients with type 2 diabetic lipid metabolism disorder", Liu Shi pointed out that phlegm and stasis play an important role in the late stage of the disease[14]. Therefore, the treatment should be to resolve phlegm and clear heat, activate blood circulation and eliminate blood stasis. Professor Liu Huaizhen makes good use of Huanglian Wenzhi Tang with blood-activating and stasis-eliminating herbs, and uses Danshen, Yujin, Paeonia lactiflora, Angelica sinensis and Chuanxiong in the treatment. Modern research has shown that Dan Shen and other drugs[9] can be used to treat obese type 2 diabetes by improving intestinal flora and regulating blood lipids. Huanglian Wenzhi Tang can improve insulin resistance through inflammatory response in order to regulate the abnormal insulin receptor signaling pathway[15]. Prevent fat and sweet before illness, and refuse to change after illness. The thirst for change is good, and the treatment of elimination should block change[16].

\section{Example of Test Case}

Patient Zhang, male, 36 years old, height $1.68 \mathrm{~m}$, weight $82 \mathrm{~kg}$, BMI: $29.05 \mathrm{~kg} / \mathrm{m}^{2}$, was first diagnosed on April 27, 2020. Complaint: recurrent dry mouth, fatigue and weakness for 2 months. The patient was found to have elevated blood glucose during physical examination 3 years ago, and was diagnosed with "type 2 diabetes" after completing relevant examinations. -The patient reported dry mouth, heavy head and body, fatigue, and chest congestion in the past two months. He has a 2-year history of dyslipidemia. The following symptoms: dry mouth, feeling heavy and drowsy, dizziness, fullness of the abdomen and stomach, weakness, lack of energy, loose stools and polyuria. The tongue is red, fat with tooth marks, yellowish moss and moist pulse. Outpatient measurement of terminal fasting glucose: $8.0 \mathrm{mmol} / \mathrm{L}$. $2 \mathrm{~h}$ postprandial glucose 10.0mmol/L. Western diagnosis: 1 . obese type 2 diabetes; 2. dyslipidemia. Traditional Chinese medicine diagnosis: spleen (damp-heat trapped spleen evidence). Treatment is to benefit Qi and strengthen the spleen, clear heat and dampness. Prescription: Radix et Rhizoma Puerariae 15g, Radix et Rhizoma Huanglian 10g, Radix Scutellariae 15g, Radix Atractylodes Macrocephalae 15g, Radix Codonopsis Pilosulae 12g, Radix et Rhizoma Saxifraga 6g, Radix Bupleurum 15g, Rhizoma Yam 30g, Radix Astragali 20g, Poria 30g, Radix Patchouli 9g, Radix et Rhizoma Glycyrrhiza 6g. 7 doses, 1 dose daily, decoction with water. The western medicine was not adjusted for the time being, and the patient was advised to eat a light diet and strengthen exercise in moderation.

On the second consultation on May 10, 2020, after taking the above medicine, dry mouth, fatigue and abdominal distension improved, but still felt dizzy, with a fat tongue with teeth marks, slightly dark texture, slightly yellowish greasy moss and slow pulse. The fasting blood glucose was measured at $7.1 \mathrm{mmol} / \mathrm{L}$ and the postprandial $2 \mathrm{~h}$ blood glucose was $10.5 \mathrm{mmol} / \mathrm{L}$. The treatment was based on the original formula with the addition of Acorus calamus 15g, Fagus sylvatica 9g, Lotus leaf $12 \mathrm{~g}$ and Salvia miltiorrhiza 15g. 21 doses were taken as before.

On June 2, 2020, the third consultation, after taking the first prescription, all the symptoms are significantly improved, self-measurement in the past 1 month fasting blood sugar about $6.2-7.1 \mathrm{mmol} / \mathrm{L}, \quad 2 \mathrm{~h}$ postprandial blood sugar 7.9-9.2mmol/L, weight decreased to $76 \mathrm{~kg}$, BMI: $26.92 \mathrm{~kg} / \mathrm{m}^{2}$. treatment above remove fried Scutellaria baicalensis, Huang Lian, add Chen Pi 12g; 14 doses, taking the same method as before. The patient insisted on light diet and exercise, and the blood glucose control was stable to the standard, and the weight was maintained at $73 \mathrm{~kg}$ to $74 \mathrm{~kg}$. At the follow-up visit for 3 months, the symptoms did not recur.

Comment: Combined with the patient's medical history and ancillary tests, a clear diagnosis of obese type 2 diabetes mellitus can be made. According to the four diagnoses, the evidence belongs to spleen deficiency and damp-heat. The patient has been over-eating thick, fatty and sweet products, eating irregularly, drinking excessively, and being tired of 
exercise, which damaged the spleen and stomach. If the spleen is deficient in transportation, phlegm and dampness are stagnant and cannot be transformed. If the spleen is deficient in transportation, phlegm and dampness are stagnant and cannot be transformed; if the spleen is deficient in spleen, weakness is seen; if the dampness and dampness are stagnant, the spleen is disturbed, the head and body are heavy and the stools are loose; if the phlegm and dampness are stagnant, heat is transformed over time; if the internal heat hurts the yin and fluid over time; if the spleen is deficient in dampness, the fluid cannot be carried to the mouth, the mouth is dry; the tongue is red, the tongue is fat and large The other tongue is red, the tongue is fat, with tooth marks on the sides, the coating is yellow and greasy, and the pulse is moistened and counted. The method should be to benefit the qi, strengthen the spleen, and clear damp-heat. The formula fried atractylodes, ginseng, poria, yam, astragalus, white lentils are all spleen-boosting drugs, can make the spleen healthy wet dispel, to achieve the purpose of supporting the right; sand, patchouli, fried atractylodes to remove dampness and full, and heavy use of Huang Lian, fried scutellaria to clear heat and dry dampness, the combination of the drugs, damp heat can be quickly dispelled; Ge Gen, roasted licorice to benefit the qi and fluids, prevent scutellaria, even over-dry injury to the spleen. The whole formula is pungent, sweet and bitter, and the herbs work together to clear damp-heat, benefit qi and strengthen the spleen; it can strengthen the spleen and earth, raise the clear yang and replenish the deficient qi of the spleen, so that dampness can be relieved, heat can be relieved, and all evidence can be removed. In the second consultation, the patient still had dizziness, so he added calamus and half ansia to increase the effect of awakening the spleen and dispelling dampness, salvia to invigorate the blood and dispel blood stasis, lotus leaf to clear heat and dampness, promote clear yang, and have a fat-reducing effect. The patient's symptoms were all removed in the third examination, removing Scutellaria baicalensis and Huang Lian to prevent the harmful effects of excessive dryness and Yin, adding Chen Pi to regulate Qi and strengthen the spleen, and adding the effect of strengthening the spleen and earth. By treating the root cause of spleen deficiency and the symptoms of dampness and heat, we not only achieved efficacy in lowering glucose, but also stabilized blood sugar, improved pancreatic function, and sustained steady weight loss. Thus, satisfactory results were obtained.

\section{Conclusion}

Based on many years of experience, Professor Liu concluded that the origin of modern obesity type 2 diabetes lies in the spleen, and damp-heat runs through the whole process of its occurrence and development; in treating obesity type 2 diabetes, Professor Liu based on the concept of "spleen deficiency and damp-heat", respecting the classics, adhering to the ancient but not the mud, advocating the method of strengthening the spleen, benefiting qi, clearing heat and relieving dampness, paying attention to the origin of the latter, taking care of the spleen and stomach, and at the same time At the same time, he also takes care of clearing heat and dampness and reconciling the three jiao, while using phlegm and eliminating stasis to prevent changes, so that the spleen is healthy, dampness is removed, and heat is resolved. Prof. Liu is good at highlighting the advantages of TCM evidence-based treatment, which brings new breakthroughs to the research of TCM prevention and treatment of diabetes, and is worthy of study by future generations.

\section{References}

[1] Clarke MA, Fetterman B, Cheung LC, et al. Epidemiologic evidence that excess body weight increases risk of cervical cancer by decreased detection of precancer[J]. J Clin Oncol, 2018, 36(12): 1184-1191.

[2] Yao L, Jiang C-X, Guo Y-Y, You C-S, Li M. Menthol 30 combined with metformin and liraglutide in the treatment of obese type 2 diabetes mellitus[J]. Western Medicine, 2020, 32(10): 1509-1512.

[3] Tai Penggang, Wang Shengshu, Wang Jianhua, Liu Miao, Yang Shanshan, He Yao. Obesity-related measurement indicators and progress of obesity measurement in elderly population[J/OL]. Journal of the PLA Medical College: 1-5[2022-01-13].

[4] Sun Guanghan, Liu Jian, Long Yan, Bao Bingxi, Zhang Ying. Research progress of Chinese medicine in the treatment of gouty arthritis[J]. Rheumatism and Arthritis, 2019, 8(08): 64-67+80.

[5] Xie Shaofeng, Huang Liji, Cao Wen, et al. Exploring the etiology and pathogenesis of obesity type 2 diabetes mellitus[J]. Chinese Journal of Basic Chinese Medicine, 2018, 24(7): 894-895.

[6] Wei Xuefang, Lin Shaobin, Xiong Hongping, et al. The effect of baicalin on islet function in diabetic rats and its mechanism[J]. Journal of Integrated Cardiovascular and Cerebrovascular Medicine, 2019, 17(19): 2933-2935.

[7] ZHANG Zexin, WU Wenfeng, XIE Dan, FANG Shuhan, XU Shaojing. Network pharmacology based combined with GEO microarray to analyze the mechanism and molecular targets of dried ginger, scutellaria, scutellaria and ginseng soup for the treatment of type 2 diabetes[J]. Journal of Guangdong University of Pharmaceutical Sciences, 2020, 36(03): 361-368

[8] LI Xin, PAN Junxian, CHEN Shiguo, et al. Research progress on the chemical composition and pharmacological effects of Pueraria lobata[J]. Chinese Journal of Food, 2017, 17(9): 189-195.

[9] Tan L, Li GX, Zhang Tie-Feng. Research progress of regulating intestinal flora in the treatment of obese type 2 diabetes mellitus[J]. World Journal of Integrated Chinese and Western Medicine, 2021, 16(08): 1565-1568.

[10] Wang M.K., Cheng L., Zhao X., Li A.L. Clinical study of Huang Lian Wen Bile Tang in the treatment of damp-heat-embedded type 2 diabetes mellitus[J]. Combined Chinese and Western Medicine Research, 2021, 13(06): 369-372.

[11] Yue Yandong, Kang Xuedong, Yang Weijie, Wu Jiayi. Analysis of the mechanism of action of pelargonium in the treatment of type 2 diabetes based on network pharmacology and molecular docking methods $[\mathrm{J}]$. Journal of Gansu University of Traditional Chinese Medicine, 2021, 38(02): 82-89.

[12] Wang Yaohui. Effectiveness and safety of San Ren Tang in the treatment of obese type 2 diabetes mellitus[J]. Clinical Journal of Traditional Chinese Medicine, 2019, 31(09): 1715-1717. 
[13] Chen Zebing, Zhou Hui, Mo Wei, et al. Clinical application of San Ren Tang in the treatment of diabetes mellitus and its complications[J]. Global Chinese Medicine, 2018, 11(8): 1291-1293.

[14] Liu Huaizhen, Tan Jiajia, Zhai YJ, Ye YF, Xu Qinghua, Song L. Relationship between TCM evidence and serum homocysteine and cystatin $\mathrm{C}$ in patients with type 2 diabetic lipid metabolism disorders[J]. Journal of Anhui University of Traditional Chinese Medicine, 2014, 33(05): 14-17.

[15] Dong Wanru, Li Han, Li Yunfeng, Wang Ning,Ma Boyan, Lu Ge Ling, Xin Xiangru, Chen Bingbing. Study on the mechanism of improving IGT by Huanglian Wenzhi Tang based on NLRP3/caspase-1/IL-1 $\beta$ and IL-18 pathways in skeletal muscle[J]. Chinese Journal of Traditional Chinese Medicine, 2021, 46(17):4480-4487.

[16] Huang Q, Tian F. Yuan. The evolution of thirst and clinical experience[J]. Journal of Zhejiang University of Traditional Chinese Medicine, 2019, 43(06): 523-526.

\section{Author Profile}

Dan Bi (1995-), female, Master's degree in Anhui University of Traditional Chinese Medicine, research interests: Chinese medicine to prevent and treat endocrine metabolic diseases.

Huaizhen Liu (1964-), female, chief physician, master of medicine, doctoral supervisor, research interests: prevention and treatment of endocrine metabolic diseases by Chinese medicine 